\title{
Geoelectrical monitoring of dissolution and precipitation reactions in a water-saturated calcareous porous medium
}

\author{
FLORE REMBERT ${ }^{1,2}$, DAMIEN JOUGNOT ${ }^{2}$, LINDA \\ LUQUOT $^{3}$ AND ROGER GUÉRIN ${ }^{2}$ \\ ${ }^{1}$ Observatoire des Sciences de 1'Univers en région Centre Val de \\ Loire \\ ${ }^{2}$ Sorbonne Université \\ ${ }^{3}$ CNRS-University of Montpellier \\ Presenting Author: flore.r@wanadoo.fr
}

Precipitation and dissolution are the main chemical processes occurring in carbonate rocks, ubiquitous at the earth subsurface. The understanding and the quantification of these processes is a major deal of reservoir exploitation for geo-resources or geological storage. Hydrogeophysical studies are based on the development of suitable geophysical techniques to monitor hydrological and biogeochemical processes non-intrusively and at low cost. Furthermore, among the existing techniques, electrical methods have already proven their ability to monitor hydrological processes and chemical reactivity. Thus, selfpotential (SP) and spectral induced polarization (SIP) methods are chosen for the study of calcite dissolution and precipitation processes. SP is a passive technique consisting in the measurement of the natural electric field generated by water fluxes and concentration gradients, through electrokinetic and electrochemical couplings, respectively, while SIP is an active method measuring the electrical complex conductivity at low frequencies (from $\mathrm{mHz}$ to $\mathrm{kHz}$ ). The electrical complex conductivity is a geophysical property whose real and imaginary components can be related to the microstructural properties of the porous medium and to the surface state of the minerals of which it is composed, respectively. However, for biogeochemical studies, SP and SIP data are still complex to interpret, due to the superposition of source mechanisms. In this study, we designed a new experimental set-up to monitor the SP and SIP signals during the dissolution and precipitation of calcite material through appropriate solution injection. We obtained clear SP response due to both chemical changes provoked by dissolution and precipitation. These SP signatures are clearly related to ionic concentration gradients, but not to water flux. Thus, to quantify the SP signal as ionic concentrations distribution in time and space, we propose a new framework based on the electrodiffusive potential for multiple ionic species coupled with 1D reactive transport simulations. This approach reproduces well the measured geo-electrical signatures, allows us to determine and predict the precise location of the reactive area and, thus, helps the SIP measurements interpretation. The framework developed for this study opens new perspectives for SP applications to characterize key processes involved in reactive transport problematic. 